\title{
Primer reporte y distribución de Delastria rosea (Pezizales: Incertae sedis) en México
}

\section{New record and distribution of Delastria rosea (Pezizales: Incertae sedis) in Mexico}

\author{
Víctor Manuel Gómez-Reyes’’3 (D), Marlene Gómez-Peraltal (D), Gonzalo Guevara Guerrero² (D)
}

1 Universidad Michoacana de San Nicolás de Hidalgo, Facultad de Biología, Herbario EBUM, Edificio R planta baja, Ciudad Universitaria, Av. Francisco J. Mújica s/n. 58030 Morelia, Michoacán, México.

2 Instituto Tecnológico de Cd. Victoria, Av. Portes Gil 1301 Pte., 87010 Cd. Victoria, Tamaulipas, México.

3 Autor para la correspondencia: vmgomez@umich.mx

Citar como:

Gómez-Reyes, V. M., M. Gómez-Peralta y G. Guevara Guerrero. 2017. Primer reporte y distribución de Delastria rosea (Pezizales: Incertae sedis) en México. Acta Botanica Mexicana 119: 139-144. DOI: http://dx.doi. org/10.21829/abm119.2017.1236

Recibido: 4 de enero de 2016.

Revisado: 29 de septiembre de 2016.

Aceptado: 24 de noviembre de 2016.

DOl:

http://dx.doi.org/10.21829/abm119.2017.1236

\section{Resumen:}

Antecedentes y Objetivos: Delastria rosea es un hongo de hábito hipogeo, se caracteriza por presentar una gleba rosa y esporas globosas con ornamentación reticulada. Se conocía hasta la fecha del norte de África, sur de Europa y California en Estados Unidos de América. El objetivo del presente trabajo es registrar D. rosea para la micobiota mexicana.

Métodos: Se realizaron recolectas siguiendo las recomendaciones para el estudio de los hongos hipogeos. El material estudiado proviene de bosque mesófilo de montaña del estado de Michoacán, México.

Resultados clave: Se describe Delastria rosea para la micobiota mexicana; se discute las semejanzas y diferencias con las descripciones europeas y norteamericanas.

Conclusiones: Se considera que los ejemplares estudiados corresponden a Delastria rosea, a pesar de las diferencias que presentan con las descripciones europeas y norteamericanas.

Palabras clave: Bosque mesófilo de montaña, hongos hipogeos, Michoacán, pseudotrufas, trufas.

\section{ABSTRACT:}

Background and Aims: Delastria rosea is a hypogeous fungus characterized by its rose gleba and globose ascospores with reticulated ornamentation. Its previously known distribution ranges from Northern Africa, Southern Europe to California in the United States of America. The aim of this study is to record $D$. rosea for the Mexican mycobiota.

Methods: The material was collected following the recommendations for the study of hypogeous fungi. It was sampled in a cloud forest in the state of Michoacán, Mexico.

Key results: Delastria rosea is described for the Mexican mycobiota and similarities and differences between the European and North American descriptions are discussed.

Conclusions: We consider that the reviewed specimens correspond to Delastria rosea, in spite of the differences presented with the European and North American descriptions.

Key words: cloud forest, hypogeous fungi, Michoacán, pseudotruffle, truffle.

\section{INTRODUCCIÓN}

El género Delastria Tul. \& C. Tul. (1843), de acuerdo con el Index fungorum, alberga dos especies, D. rosea Tul. \& C. Tul., (1843) y D. supernova A. Paz \& C. Lavoise (2014). Trappe et al. (1979) ubicó a dicho género dentro de la familia Terfeziaceae por presentar esporas globosas y una ornamentación reticulada; sin embargo, aclara que las relaciones entre Delastria y el resto de los integrantes de la familia no son claras. Castellano et al. (2004) ubicaron al género en la familia Tuberaceae. Actualmente, de acuerdo con Lumbsch y Huhndorf (2007) y la base de datos Index fungorum, el género Delastria se ubica como Incertae sedis dentro 
del orden Pezizales (Pezizomycetes, Ascomycota). Læssøe y Hansen (2007) mencionaron la necesidad de realizar estudios de ADN para aclarar la posición de este taxon.

Por más de 170 años, el género Delastria se mantuvo como monoespecífico, con una sola especie descrita, D. rosea, la cual se caracteriza por presentar ascocarpos subglobosos, turbinados a irregulares, de 10 a $30 \mathrm{~mm}$ de diámetro, blancos, aterciopelados o tomentosos; el peridio compuesto de hifas entremezcladas con células infladas isodiamétricas; y la gleba compuesta por una área fértil rosa y con numerosas venas infértiles blancas. Microscópicamente se caracteriza por las esporas globosas de 23 a $35 \mu \mathrm{m}$, pared lisa cuando inmaduras, pero al madurar desarrollan una ornamentación retículo alveolada con espinas de hasta $3 \mu \mathrm{m}$ de alto. La distribución de la especie se reporta para el sur de Europa y el norte de África (Kirk et al., 2008), mientras que para Norte América fue citada por Harkness (1899) y Gilkey $(1916,1954)$ del estado de California en Estados Unidos de América.

La segunda especie del género, D. supernova, fue descrita por Paz y Lavoise (2014) con material procedente del norte de la península Ibérica. Esta especie se caracteriza por presentar un peridio de 180-250 $\mu \mathrm{m}$ en dos capas, una epicutis de 7-12 $\mu \mathrm{m}$ compuesta de hifas ramificadas, de pared delgada y una hipocutis compuesta de células subglobosas 25-35 × 20-26 $\mu \mathrm{m}$, de pared gruesa de 2 a $3 \mu \mathrm{m}$, mientras que las esporas son ampliamente elipsoides a subglobosas de 24-26 × 20-22 $\mu \mathrm{m}$, con una ornamentación retículo alveolada, con espinas de 2-3 $\mu \mathrm{m}$ de alto.

El objetivo del presente trabajo es registrar y describir Delastria rosea para México, así como presentar la nueva distribución del taxon, además de continuar con el reporte de los hongos con hábito hipogeo del centro de México.

\section{Materiales y MÉtOdOS}

El material recolectado se estudió siguiendo las recomendaciones de Castellano et al. (1989) y Pegler et al. (1993). Los colores de los ejemplares en fresco se basaron en la carta de identificación de colores del Royal Botanic Gar- den Edinburgh (1969). Para examinar las características microscópicas, se realizaron cortes a mano de algunas estructuras útiles en taxonomía como ascas, ascosporas, trama himenial y peridio, principalmente, los cuales fueron montados en $\mathrm{KOH}$ al 5\%, agua y Reactivo de Melzer. Se consideraron 30 medidas en objetivo de 100× utilizando un microscopio Motic BA300 (Hong Kong, China) con cámara digital Moticam $2300 \mathrm{y}$ el software Motic Image Plus 2.0 (Motic, 2004). Los ejemplares deshidratados se encuentran depositados en la Colección de macromicetos del Herbario EBUM de la Facultad de Biología de la Universidad Michoacana de San Nicolás de Hidalgo y con un duplicado en el Herbario ITCV José Castillo Tovar del Instituto Tecnológico de Ciudad Victoria.

\section{Resultados}

\section{Descripción}

Delastria rosea Tul. \& C. Tul., Annls Sci. Nat., Bot., sér. 2. 19: 379. 1843.

$\equiv$ Terfezia rosea (Tul. \& C. Tul.) Torrend, Bolm Soc. Portug. Ciênc. Nat. 1: 180. 1908. Figs. 1A-E.

Ascoma de 8-10 × 6-9 mm diámetro, globoso a subgloboso, lobulado o algo irregular; peridio blanco (2B) a blanco amarillento (3C, 4D), superficie pubescente con partículas de suelo y materia orgánica adherido, el peridio se continúa hacia la gleba formando venas internas, no se observó cambio de color con el maltrato, reacción positiva del peridio a $\mathrm{KOH} 5 \%$ de blanco amarillento a azul oscuro o negruzco; gleba sólida, con áreas redondeadas fértiles de color rosa pálido (39), rosa oscuro, rojo pálido (41), con numerosas venas blancas que se unen al peridio, que dan la apariencia de formar "paquetes" redondos al interior; olor y sabor no registrados, peridio de 250-350 $\mu \mathrm{m}$ de grosor en dos capas, una constituida por un epicutis $10-20 \mu \mathrm{m}$ de grosor, compuesto de hifas postradas con terminaciones erectas, donde se distinguen tres tipos de hifas: las más abundantes corresponden a hifas de pared delgada, septadas, ramificadas, hialinas en 

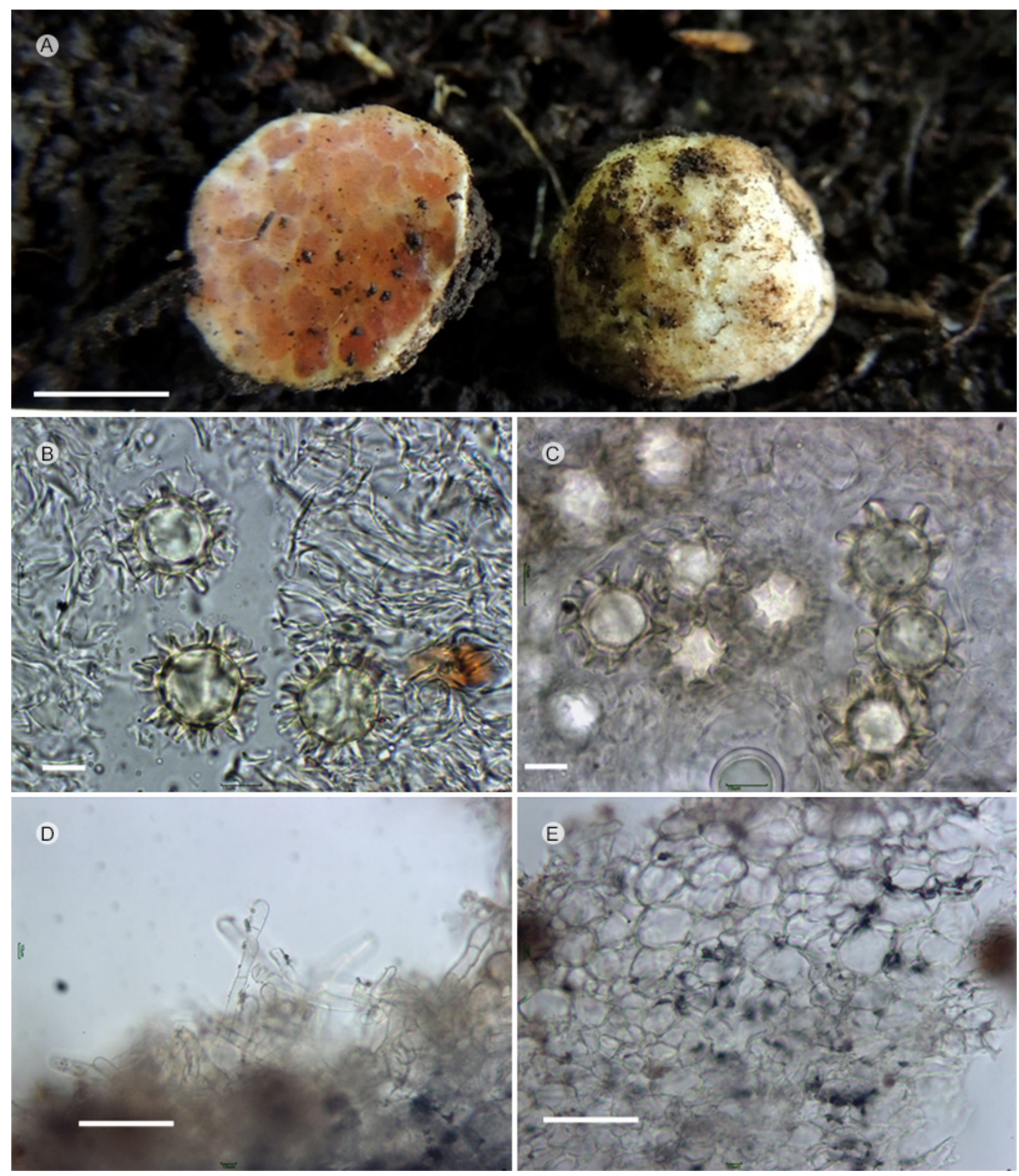

Figura 1: Delastria rosea Tul. \& C. Tul. (V. M. Gómez-Reyes 892). A. ascoma, (barra=5 mm); B y C. ascosporas y asca con ascosporas (barra=10 $\mu \mathrm{m}$ ); D y E. peridio (barra $=50 \mu \mathrm{m}$ ); D. células claviformes terminales en epicutis; E. células pseudoparenquimatosas del subcutis. Fotos: V.M. Gómez-Reyes. 
$\mathrm{KOH}$, de 4-14 $\mu \mathrm{m}$ de ancho con el ápice redondeado y con partículas incrustadas en las paredes, de manera esporádica se presentan hifas de pared gruesa, septadas, azul oscuro en $\mathrm{KOH}$, de 1.5-3.5 $\mu \mathrm{m}$ de ancho, aciculiformes o claviformes, el tercer tipo de hifa corresponde a hifas de pared gruesa, septadas, de color café en $\mathrm{KOH}$, de 4-7 $\mu \mathrm{m}$ de ancho; la segunda capa, el subcutis presenta arreglo pseudoparenquimatoso y se compone de hifas con células isodiamétricas que van de (8)10-35(50) × (6)9-30(35) $\mu \mathrm{m}$, de pared gruesa, $2 \mu \mathrm{m}$ de grosor; gleba compuesta de hifas septadas, hialinas en $\mathrm{KOH}$, entrelazadas de pared delgada, de 3-8 $\mu \mathrm{m}$ de ancho y células globosas isodiamétricas entremezcladas de 5-12 × 10-24 $\mu \mathrm{m}$; ascas de (80)100-135 × (30)40-55 $\mu \mathrm{m}$, hialinas, clavadas, de pared delgada con un corto pedúnculo, 3(-4) esporas por asca; ascosporas de 17-26 × 17-24 $\mu \mathrm{m}$ sin la ornamentación, globosas a subglobosas $(\mathrm{Q}=1-1.12$, promedio 1.04$)$, de jóvenes lisas, cuando maduras desarrollan un exosporio retículo alveolado, amarillo verdoso en $\mathrm{KOH}$, inamiloides, alveolos irregulares, formado de 2-4 celdas, mientras que la parte externa forma espinas piramidales, 4-7 $\mu \mathrm{m}$ de alto, 3-5 $\mu \mathrm{m}$ en la base, en algunas se observa el ápice curvo.

Habito, hábitat y temporada: hipogeo, solitario, bajo Carpinus caroliniana Walter en bosque mesófilo de montaña. Se ha colectado de julio a septiembre.

Material revisado: MÉXICO. Michoacán, Uruapan, La Alberca, Toreo el Alto, 19²9'29.65"N, $102^{\circ} 00^{\prime} 18.25^{\prime \prime O}, 2015$ m, 17.VII.2015, V. M. GómezReyes 892 (EBUM!), 19²9'27.28"N, 10200'17.10"O, 2018 m 11.IX.2015, A. M. Ortega Gómez s.n. (EBUM!, ITCV!).

\section{Discusión}

El género Delastria se suma a la lista de ascomicetos con hábito hipogeo citados para México. Otros géneros de este grupo presentes en el país son Carbomyces Gilkey, Elaphomyces Nees, Genea Vittad., Geopora Harnk., Gilkeya M.E. Sm., Trappe \& Rizzo, Hydnobolites Tul. \& C. Tul.,
Pachyphloeus Tul. \& C. Tul., Mattirolomyces E. Fisch. y Tuber P. Micheli ex F.H. Wigg. (Trappe y Guzmán, 1971; Trappe et al., 1979; Cázares et al., 1992; Smith et al., 2006; Trappe y Cázares, 2006; Guevara et al., 2008; 2012; 2013; 2015; Healy et al., 2009; Kovács et al., 2011; Castellano et al., 2012; Gómez-Reyes et al., 2012; Moreno et al., 2012). En conjunto se han citado 41 especies de ascomicetos para México, incluyendo este nuevo registro de Delastria rosea.

El género Delastria se distingue de otros géneros de ascomicetos con hábito hipogeo por la gleba con áreas redondeadas de color rosa y venas blancas, mientras que el peridio es evanescente en la madurez y pubescente en ejemplares jóvenes. La distribución conocida de las dos especies descritas de Delastria abarca del Norte de África al sur de Europa (Montecchi y Sarasini, 2000) y California en Estados Unidos de América (Harkness, 1899; Gilkey, 1916; 1939; 1954).

Delastria rosea se distingue de D. supernova por el tamaño de las esporas y el largo de las espinas del exosporio. Delastria rosea, de acuerdo con Montecchini y Sarasini (2000) y Calonge et al. (1985), presenta esporas de 20 a $35 \mu \mathrm{m}$ y espinas hasta de 3-5 $\mu \mathrm{m}$ de alto, en ambos casos con base en material europeo. Gilkey (1939), con base en material de California, menciona que las esporas son de $22-30 \mu \mathrm{m}$ y las espinas hasta de $5 \mu \mathrm{m}$, mientras que $D$. supernova presenta esporas de 24 a $36 \mu \mathrm{m}$ y las espinas más cortas de 2-3 $\mu \mathrm{m}$.

Los ejemplares mexicanos presentan algunas diferencias con las descripciones europeas y norteamericanas (Calonge et al., 1985; Gilkey, 1939; Montecchini y Sarasini, 2000), principalmente en la medida de las esporas. En conjunto las describen de 20-35 $\mu \mathrm{m}$ con espinas hasta de $5 \mu \mathrm{m}$, mientras que las colectas mexicanas presentan esporas más pequeñas de 17-25 $\mu \mathrm{m}$ y espinas de 5-7 $\mu \mathrm{m}$ de alto. Otra diferencia son los hábitats; los ejemplares mexicanos se recolectaron en bosque mesófilo de montaña y en posible asociación con Carpinus caroliniana, mientras que $D$. rosea en Europa se encuentra asociada con arbustos del mediterráneo y con Pinus pinaster Aiton en suelo arenoso. 
Los ejemplares mexicanos de Delastria rosea, a pesar de las diferencias con las descripciones de Europa, presentan suficiente similitud por lo que consideramos que efectivamente pertenecen a esta especie. Sin embargo, estudios de ADN en el futuro podrán confirmar esta identificación.

\section{CONTRIBUCIÓN DE LOS AUTORES}

VMGR recolectó y revisó el material; además, escribió el primer borrador del artículo; MGP y GGG revisaron el material y concordaron en la identificación taxonómica, además corrigieron y contribuyeron sustancialmente en la versión final. VMGR, MGP y GGG contribuyeron en la discusión, revisión y aprobación del manuscrito final.

\section{FINANCIAMIENTO}

VMGR agradece al Consejo Nacional de Ciencia y Tecnología por el apoyo otorgado para la estancia posdoctoral Vinculada al Fortalecimiento de la Calidad del Posgrado Nacional. MGP agradece y reconoce el apoyo de la Coordinación de la Investigación Científica de la Universidad Michoacana de San Nicolás de Hidalgo a través del proyecto 8.15 "Catálogo de hongos hipogeos (trufas y pseudotrufas) del Sistema Volcánico Transversal de Michoacán". GGG agradece al Programa de Mejoramiento del Profesorado, Dirección General de Educación Tecnológica y Consejo Nacional de Ciencia y Tecnología por el apoyo brindado en esta investigación.

\section{AgRADECIMIENTOS}

Se agradece a los dos revisores anónimos por los comentarios y observaciones que contribuyeron al manuscrito.

\section{LITERATURA CITADA}

Calonge, F. D., A. Rocabruna, M. Tabarés y N. B. Rodríguez. 1985. Nuevos datos sobre los hongos hipogeos de España. II. Géneros Balsamia, Delastria y Genea, novedades para el catálogo español. Boletín de la Sociedad Micológica 9: 57-64.
Castellano, M. A., J. M. Trappe, Z. Maser y C. Maser. 1989. Keys to spores of the genera of hypogeous fungi of North temperate forests with special reference to animal mycophagy. Mad River Press. Eureka, USA. 186 pp.

Castellano, M. A., J. M. Trappe y D. L. Luoma. 2004. Secuestre Fungi. In: Mueller, G. M, G. F. Bills and M. S. Foster (eds.). Biodiversity of Fungi, Inventory and Monitoring Methods. Elsevier Academic Press. London, UK. Pp. 197-213.

Castellano, M. A., G. Guevara, J. García y J. M. Trappe. 2012. Elaphomyces appalachiensis and E. verruculosus sp. nov. (Ascomycota, Eurotiales, Elaphomycetaceae) from Eastern North America. Revista Mexicana de Micología 35: 17-22. Cázares, E., J. García, J. Castillo y J. M. Trappe. 1992. Hypogeous fungi from Northern Mexico. Mycologia 84(3): 341-359. DOI: http://dx.doi.org/10.2307/3760186

Gilkey, H. M. 1916. A revision of the Tuberales of California. University of California Publications in Botany 6(11): 275-356.

Gilkey, H. M. 1939. Tuberales of North America. Oregon State College. Studies in Botany 1: 1-39.

Gilkey, H. M. 1954. Tuberales. North American Flora. Series II, Part I. The New York Botanical Garden. New York, USA. 36 pp.

Gómez-Reyes, V. M., I. R. Hernández-Salmeron, A. Terrón-Alfonso y G. Guevara. 2012. Estudio taxonómico de Elaphomyces spp. (Ascomycota, Eurotiales, Elaphomycetaceae) de Michoacán, México. Revista Mexicana de Micología 36: 57-62.

Guevara, G., G. Bonito, E. Cázares, J. Rodríguez, R. Vilgalys y J. M. Trappe. 2008. Tuber regimontanum, new species of truffle from Mexico. Revista Mexicana de Micología 26: $17-20$.

Guevara, G., B. Stielow, H. Tamm, E. Cázares-González y M. Göker. 2012. Genea mexicana, sp. nov., and Geopora tolucana sp. nov., new hypogeous Pyronemataceae from Mexico, and the taxonomy of Geopora reevaluated. Mycological Progress 11: 711-724. DOI: http://dx.doi. org/10.1007/s11557-011-0781-y

Guevara, G., G. Bonito y E. Cázares. 2013. Revisión del género Tuber (Tuberaceae: Pezizales) de México. Revista 
Mexicana de Biodiversidad S39-S49. DOI: http://dx.doi. org/10.7550/rmb.31981

Guevara, G., G. Bonito, E. Cázares-González, R. Healy, R. Vilgalys y J. Trappe. 2015. Novel Tuber spp. (Tuberaceae, Pezizales) in the Puberulum Group from Mexico. Ascomycete.org 7(6): 367-374.

Harkness, H. W. 1899. Californian Hypogæous fungi. Proceedings of the California Academy of Sciences, Serie 3, Botany I(8): 241-293.

Healy, R. A., G. Bonito y G. Guevara. 2009. The truffle genus Pachyphloeus in the U.S. and Mexico: phylogenetic analysis and a new species. Mycotaxon 107: 61-71. DOI: http://dx.doi.org/10.5248/107.61

Index fungorum. 2016. Index fungorum base de datos. http:// www.indexfungorum.org/Names/Names.asp. (consultado octubre de 2016).

Kirk, P. M., P. F. Cannon, D. W. Minter y J. A. Stalpers (eds.). 2008. Ainsworth \& Bisby's Dictionary of the Fungi. CABI. Wallinford, UK. 771 pp.

Kovács, G. M., J. M. Trappe, A. M. Alsheikh, K. Hansen, R. A. Healy y P. Vági. 2011. Terfezia disappears from the American truffle mycota as two new genera and Mattirolomyces species emerge. Mycologia 103(4): 831-840. DOI: http://dx.doi.org/10.3852/10-273

Læssøe, T. y K. Hansen. 2007. Truffle trouble: what happened to the Tuberales? Mycological Research 111: 1075-1099. DOI: http://dx.doi.org/10.1016/j.mycres.2007.08.004

Lumbsch, H. T. y S. M. Huhndorf. 2007. Notes on ascomycete systematics. Nos. 4408-4750. Myconet 13: 59-99.

Montecchi, A. y M. Sarasini. 2000. Funghi ipogei d'Europa. Associazione Micologica Bresadola, Fondazione Centro Studi Micologici. Vicenza, Italia. 714 pp.
Moreno, G., M. Lizárraga, M. Esqueda, R. Galán y P. Alvarado. New records of little-known species of Carbomyces (Carbomycetaceae, Ascomycota). Mycotaxon 120: 89-98. DOI: http://dx.doi.org/10.5248/120.89

Motic. 2004. Motic Image Plus 2.0. Motic China group. Co., Ltd. Hong Kong, China. http://www.motic.com.

Paz, A. y C. Lavoise. 2014. Propuesta de dos nuevas especies de hongos hipogeos y una primera cita para la Península Ibérica. Boletín Micológico de FAMCAL 8: 71-86.

Pegler, D. N., B. M. Spooner y T. W. K. Young. 1993. British truffles, a revision of British hypogeous fungi. Royal Botanic Gardens, Kew, UK. 242 pp.

Royal Botanic Garden Edinburgh. 1969. Flora of British Fungi, Color Identification Chart. 5 ed. Scotland, UK.

Smith, M. E., J. M. Trappe y D. M. Rizzo. 2006. Genea, Genabea and Gilkeya gen. nov.: ascomata and ectomycorrhiza formation in Quercus Woodland. Mycologia 98(5): 699716. DOI: http://dx.doi.org/10.3852/mycologia.98.5.699

Trappe, J. M. y G. Guzmán. 1971. Notes on some hypogeous fungi from Mexico. Mycologia 63: 317-332. DOI: http:// dx.doi.org/10.2307/3757764

Trappe, J. M., G. Guzmán G. y C. Vázquez-Salinas. 1979. Observaciones sobre la identificación, distribución y usos de los hongos del género Elaphomyces en México. Boletín de la Sociedad Mexicana de Micología 13: 145-150.

Trappe, J. M. y E. Cázares. 2006. Tuber guzmanii, a new truffle from Southern México. International Journal of Medicinal Mushrooms 8(3): 279-282. DOI: http://dx.doi. org/10.1615/IntJMedMushr.v8.i3.90 